\title{
Evaluation of 2-methoxyestradiol serum levels as a potential prognostic marker in malignant melanoma
}

\author{
IGOR HRGOVIC $^{1,2^{*}}$, JOHANNES KLEEMANN $^{1 *}$, MONIKA DOLL $^{1}$, CARMEN LOQUAI $^{3}$, \\ FLORIAN WEID $^{3}$, FRANK LOUWEN ${ }^{4}$, NADJA ZOELLER ${ }^{1}$, STEFAN KIPPENBERGER $^{1}$, \\ ROLAND KAUFMANN $^{1}$ and MARKUS MEISSNER ${ }^{1}$
}

${ }^{1}$ Department of Dermatology, Venereology and Allergology, Goethe University, D-60590 Frankfurt;

${ }^{2}$ Department of Dermatology and Allergology, University Medical Centre Giessen, Justus Liebig University, D-35385 Giessen;

${ }^{3}$ Department of Dermatology, University Medical Centre Mainz, D-55131 Mainz;

${ }^{4}$ Department of Gynaecology and Obstetrics, Goethe University, D-60590 Frankfurt, Germany

Received July 13, 2020; Accepted April 1, 2021

DOI: $10.3892 / \mathrm{mco} .2021 .2303$

\begin{abstract}
Experimental findings indicated that 2-methoxyestradiol (2-ME), an endogenous metabolite of $17 \beta$-estradiol, may exhibit anti-tumorigenic properties in various types of tumour, such as melanoma and endometrial carcinoma. In patients with endometrial cancer, the serum levels of 2-ME are decreased compared with those in healthy controls, and this finding has been associated with a poor outcome. The aim of the present study was to examine whether the serum levels of 2-ME are decreased in patients with melanoma, and whether this decrease may be correlated with disease stage and, therefore, serve as a prognostic indicator. ELISA was used to detect serum levels of 2-ME in patients with stage I-IV malignant melanoma (MM). A cohort of 78 patients with MM was analysed, along with 25 healthy controls, among whom 15 were women in the second trimester of pregnancy (positive control). As expected, significantly elevated levels of serum 2-ME were observed in pregnant control patients compared with those in patients with MM and healthy controls. There was no observed correlation between 2-ME serum levels in patients with MM and disease stage, tumour thickness, lactate dehydrogenase or S100 calcium-binding protein B levels. In addition, the 2-ME levels of patients with MM did not differ significantly from those of normal healthy controls. Overall, the findings of the present study indicated that the 2-ME serum levels in patients with MM were not decreased, and
\end{abstract}

Correspondence to: Professor Markus Meissner, Department of Dermatology, Venereology and Allergology, Goethe University, Theodor Stern Kai 7, D-60590 Frankfurt, Germany

E-mail: markus.meissner@kgu.de

*Contributed equally

Key words: 2-methoxyestradiol, melanoma, lactate dehydrogenase, stage, S100 calcium-binding protein B, prognostic factor there was no correlation with early- or advanced-stage disease. Therefore, in contrast to published results on endometrial cancer, endogenous serum 2-ME levels in MM were not found to be correlated with tumour stage and did not appear to be a suitable prognostic factor in MM.

\section{Introduction}

Human malignant melanoma (MM) is a life-threatening and highly aggressive tumour with a pronounced propensity to metastasize. The incidence and mortality rates of MM have been increasing worldwide. In Germany, there were an estimated 21,410 new MM cases and 3,042 deaths in 2016, whereas the reported $\mathrm{MM}$ incidence among women and men was reported to increase by $>4 \%$ annually between 2003 and 2013 (1). Although the 5-year survival rate for early-stage MM is $>90 \%$ and drug therapy for metastatic MM has rapidly progressed in recent years, the 3-year overall survival decreases to $<60 \%$ for advanced-stage MM with distant metastasis (2). Therefore, it is crucial to identify diagnostic markers to enable early identification of disease progression, which may be pivotal in improving survival outcomes for patients with advanced-stage MM. However, only a few clinically useful serological markers are currently available, and the prognostic outcome is mainly determined by tumour thickness, lymph node involvement, ulceration status of the excised primary tumour and identification of distant metastasis. To date, the only biomarker used in the American Joint Committee on Cancer (AJCC) staging system is lactate dehydrogenase (LDH), the most validated and strongest independent prognostic factor for MM with distant metastasis (3). In addition to LDH, S100 calcium-binding protein $\mathrm{B}(\mathrm{S} 100 \mathrm{~B})$ is another prognostic and diagnostic serum marker in MM patients with distant metastasis for monitoring tumour response to treatment and identifying recurrent disease (4). Several other diagnostic serum factors have been investigated in MM with distant disease, such as melanoma inhibitory activity protein, galectin-3, vascular endothelial growth factor (VEGF) and matrix metalloproteinase-9, but none have reached clinical relevance (5). Therefore, there are 
currently no validated serum markers for identifying MM progression at its earlier stages.

2-Methoxyestradiol (2-ME) is an endogenous metabolite of $17 \beta$-estradiol, which is generated by catechol-O-methyltransferase (6). Recent studies have revealed that the serum levels of 2-ME are low in non-pregnant women and physiologically increase during pregnancy, reaching a maximum during the last trimester, whereas 2-ME level is suppressed in women with preeclampsia (7). A number of studies suggested that 2-ME is critical for placental homeostasis by regulation of cytotrophoblast differentiation and oxygen tension during normal pregnancy, whereas impaired 2-ME levels have been implicated in the pathophysiology of preeclampsia (8). Interestingly, beside these physiological effects in pregnancy, it has been found that 2-ME exerts anticancer effects by inducing growth arrest or caspase-dependent and -independent apoptosis in a number of tumour cell lines (e.g., melanoma, bladder cancer, leukaemia and ovarian cancer) in vitro and in mouse experiments in vivo (9-12). Additionally, 2-ME has been shown to inhibit angiogenesis by modulating angiogenesis-related genes, such as hypoxia inducible factor-1 $\alpha(\operatorname{HIF}-1 \alpha)$ and $\operatorname{VEGF}(13,14)$. Therefore, clinical trials with 2-ME in cancer treatment are underway (15). Furthermore, it has been found that 2-ME levels were reduced in patients with endometrial cancer compared with those in healthy controls (16). In melanoma cell 2-ME was able to inhibit cell growth and to induce apoptosis (17).

In view of this data and with the knowledge, that angiogenesis plays a crucial role in melanoma progression it was hypothesized that 2-ME serum levels could be reduced in patients with malignant melanoma. Therefore, the aim of the present pilot study was to analyse 2-ME serum levels in melanoma patients compared to healthy volunteers (negative control) and pregnant woman (positive control). We analysed whether 2-ME serum levels of patients with MM are correlated with the tumour stage and if they can be used as prognostic/diagnostic serum markers during disease progression. The evaluation of 2-ME as a treatment option in melanoma patients was not addressed in this study.

\section{Materials and methods}

Ethics statement. All patients and healthy controls provided written informed consent prior to enrolment and publication of any data according to the principles outlined in the Declaration of Helsinki. The study protocol was approved by the Institutional Ethics Committee Frankfurt (ethic vote SDO-01-2013) and Mainz [ethic vote 837.270.14 (9509)].

Study population. A total of 78 treated and subsequently followed-up patients with histologically confirmed stage I-IV MM recruited between May 2014 and August 2016 from the Departments of Dermatology of the University Hospitals in Frankfurt and Mainz (Germany) were included in the present study. The patients were staged according to the 8th edition of the AJCC staging system (3). The following data on the characteristics of each patient were collected where available: Sex, age, Breslow's tumour thickness, disease stage according to the AJCC staging system, serum LDH and S100B levels. Details on the clinical and histopathological characteristics of all enrolled patients are provided in Table I.
Control samples. Serum samples were collected from 10 healthy blood donors ( 4 women and 6 men with a mean age of 41 years; range, 30-53 years) at the Department of Dermatology of the University Hospital Frankfurt (Germany) and used as controls for this study. Additionally, serum samples were collected from 15 healthy women in the second trimester of pregnancy, (mean age, 32 years; range, 26-40 years) at the Department of Gynaecology and Obstetrics of the University Hospital Frankfurt (Germany) and used as positive controls for 2-ME serum levels.

Sample collection. Venous blood samples were obtained and separated within $1 \mathrm{~h}$ by centrifugation at $1,000 \mathrm{x}$ g for $15 \mathrm{~min}$ at $4^{\circ} \mathrm{C}$. All collected serum samples were stored at $-80^{\circ} \mathrm{C}$ until analysis. Lipaemic or haemolysed samples were eliminated from the study.

Measurement of 2-ME serum levels. Serum samples were assayed with the 2-ME ELISA kit cat. no. 582261 (Cayman Chemical Company) according to the manufacturer's instructions. The optical density was measured using an ELISA reader (ELISA-Reader ASYS Expert 96; Deelux Labortechnik GmbH).

Statistical analysis. Statistical analyses were performed using Microsoft Excel and SigmaPlot 12.0 software (Systat Software, Inc.). $\mathrm{P}<0.05$ was considered to indicate statistically significant differences. To test for normality distribution, the Shapiro-Wilk test was performed. The Wilcoxon Mann-Whitney U test was used to test for differences between two groups. For differences between more than two groups, the Kruskal-Wallis test was applied. Subsequently, to test for differences between groups, Dunn's post hoc analysis was employed. Correlation coefficients were determined by Spearman's correlation analysis.

\section{Results}

Comparison of 2-ME serum levels between controls and patients with MM. First, the 2-ME serum levels of the healthy volunteers (negative control group) and healthy women in second trimester of pregnancy (positive control group) were evaluated at baseline. As expected, it was observed that healthy pregnant women exhibited significantly higher mean concentrations of 2-ME in comparison with the healthy control group ( 258.4 vs. $138.7 \mathrm{pg} / \mathrm{ml}$, respectively; $\mathrm{P}<0.05$ ). Furthermore, the 2-ME serum levels of the 78 recruited MM patients were evaluated. No significant difference in the serum levels of 2-ME was observed between MM patients and the healthy control group (121.7 vs. $138.7 \mathrm{pg} / \mathrm{ml}$, respectively; $\mathrm{P}>0.05$ ), whereas the serum levels of healthy pregnant women (positive control) were significantly higher compared with those of patients with MM (258.4 vs. $121.7 \mathrm{pg} / \mathrm{ml}$, respectively; $\mathrm{P}<0.05)$. Taken together, these findings demonstrated that serum 2-ME is not suitable for distinguishing between MM patients and healthy controls (Fig. 1).

Association between serum 2-ME levels and clinical prognostic factors in $M M$. Further analysis revealed that the serum levels of 2-ME were not correlated with tumour thickness in MM patients $(r=-0.188, P>0.05)$. Evaluation of 2-ME serum levels in relation to the tumour stages of the TNM classification (T0-T4) showed no significant differences 
Table I. Clinicopathological characteristics of patients with melanoma $(\mathrm{n}=78)$.

\begin{tabular}{lc}
\hline Clinical variable & Value \\
\hline Sex, $\mathrm{n}$ & \\
Male & 52 \\
Female & 26 \\
Median age (range), years & $61(30-87)$ \\
Breslow thickness of primary tumor, $\mathrm{n}$ & \\
Tx & 1 \\
T0 (no evidence of primary tumor) & 11 \\
T1 $(\leq 1 \mathrm{~mm})$ & \\
T1a $(<0.8 \mathrm{~mm}$, not ulcerated $)$ & 7 \\
T1b $(\geq 0.8 \mathrm{~mm})$ & 5 \\
T2 $(1.1-2.0 \mathrm{~mm})$ & 21 \\
T3 $(2.1-4.0 \mathrm{~mm})$ & 22 \\
T4 $(>4 \mathrm{~mm})$ & 11 \\
Clinical stage, $\mathrm{n}$ & \\
I & 7 \\
II & 12 \\
III & 25 \\
IV & 34 \\
Lactate dehydrogenase, $\mathrm{n}$ & \\
$<248 \mathrm{U} / 1$ & \\
$\geq 248 \mathrm{U} / \mathrm{l}$ & 46 \\
Unknown & 24 \\
S100 calcium-binding protein B, $\mathrm{n}$ & 8 \\
$<0.105 \mu \mathrm{g} / \mathrm{l}$ & \\
$\geq 0.105 \mu \mathrm{g} / 1$ & 57 \\
Unknown & 13 \\
\hline
\end{tabular}

between groups $(\mathrm{P}>0.05)$ (Table II). Further evaluation of tumour substages was not performed due to the limited sample size. Furthermore, a possible association between the serum levels of 2-ME and S100B in the recruited MM patients was investigated. It was observed that 2-ME could not differentiate between patients with normal S100B levels $(\leq 0.105 \mu \mathrm{g} / \mathrm{l}$; $\mathrm{n}=57)$ and those with elevated S100B levels $(\geq 0.105 \mu \mathrm{g} / \mathrm{l}$; $\mathrm{n}=13)(124.4$ vs. $111.9 \mathrm{pg} / \mathrm{ml}$, respectively; $\mathrm{r}=-0.0485 ; \mathrm{P}>0.05)$. Additionally, a possible correlation between the serum levels of $2-\mathrm{ME}$ and $\mathrm{LDH}$ was investigated in the patients. The results of the analysis demonstrated that 2-ME was unable to distinguish between MM patients with normal serum LDH levels $(<248 \mathrm{U} / 1 ; \mathrm{n}=46)$ and those with elevated serum LDH levels $(\geq 248 \mathrm{U} / \mathrm{l} ; \mathrm{n}=24)(128.1$ vs. $110.5 \mathrm{pg} / \mathrm{ml}$, respectively; $\mathrm{r}=-0.0478 ; \mathrm{P}>0.05)$. Finally, the 2-ME serum level distribution was analysed in MM patients with AJCC stage I $(113.6 \mathrm{pg} / \mathrm{ml}$; $\mathrm{n}=8)$, II (122.2 pg/ml; $\mathrm{n}=11)$, III (132.2 pg/ml; $\mathrm{n}=25)$ and IV $(116.8 \mathrm{pg} / \mathrm{ml} ; \mathrm{n}=34)$. The results revealed no significant correlation between the concentration of 2-ME and AJCC stage $(\mathrm{r}=-0.0417 ; \mathrm{P}>0.05)$. Therefore, none of the known clinical parameters, such as Breslow tumour thickness, S100B and LDH serum levels and AJCC stage, were found to be significantly correlated with the serum 2-ME levels in patients with MM (Table II).

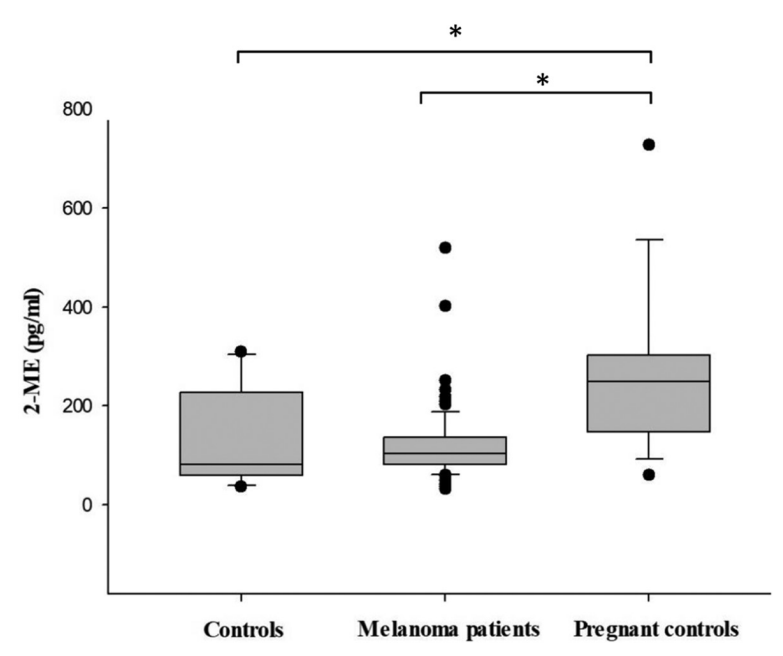

Figure 1. Measurement of 2-ME serum levels in pregnant controls $(n=15)$, non-pregnant controls $(\mathrm{n}=10)$ and patients with malignant melanoma $(\mathrm{n}=78)$. The central box indicates the upper and lower percentiles (from $\mathrm{P}_{25}$ to $\mathrm{P}_{75}$ ), whereas the middle line represents the median. The upper and lower horizontal bars extend from the minimum to the maximum value; outliers are indicated by black dots. ${ }^{*} \mathrm{P}<0.05$. 2-ME, 2-methoxyestradiol.

\section{Discussion}

MM is a potentially life-threatening disease with a poor prognosis in the advanced stages (2). Improvement of survival outcomes of patients with MM largely depends on early detection. To date, there are only two clinically approved serum markers for the monitoring of patients with MM: S100B and $\mathrm{LDH}$. Unfortunately, the prognostic value of both markers is limited and therefore, there is a great need to identify new prognostic biomarkers.

From preclinical data, 2-ME appeared to be a promising molecule to investigate as a prognostic marker in melanoma. It has been demonstrated that 2-ME exerts anti-angiogenic effects through downregulation of VEGF and HIF-1 $\alpha$ and induces apoptosis in endothelial cells $(13,14,18)$. It has been reported that 2-ME inhibits cell proliferation by inducing apoptosis and promoting $\mathrm{G} 2 / \mathrm{M}$ phase arrest of $\mathrm{MM}$ cells $(11,17)$. Additionally, exogenic applied 2-ME has been shown to be effective against MM growth, independent of the BRAF mutational status, and significantly decreased cell proliferation in a 3D skin reconstruction model (11). Based on these data and findings from a recent study on patients with endometrial carcinoma who exhibited reduced endogenous levels of 2-ME compared to healthy subjects, we decided to assess 2-ME serum levels in patients with MM compared to healthy volunteers (negative control) and pregnant woman (positive control) (16). Our intention was to evaluate the potential of endogenously generated 2-ME, as a prognostic serum marker and therefore patients were not exposed to exogenous 2-ME. As expected, the 2-ME serum levels were elevated in pregnant healthy women compared to healthy volunteers and MM patients. The 2-ME serum levels of the MM patients were slightly, but not significantly, lower compared with those of healthy controls. In the present study, 2-ME levels were not found to be correlated with any of the analysed prognostic relevant clinicopathological parameters, such as tumour thickness, AJCC stage, S100B and LDH serum levels. 
Table II. 2-ME levels and the clinical characteristics of patients with melanoma.

\begin{tabular}{|c|c|c|c|c|}
\hline Clinical characteristics & Cases, $\mathrm{n}$ & $\begin{array}{l}\text { Mean 2-ME serum } \\
\text { levels, pg/ml }\end{array}$ & P-value & $\begin{array}{c}\text { Spearman correlation } \\
\text { coefficient }\end{array}$ \\
\hline Patients & & & $<0.05^{\mathrm{a}, \mathrm{b}}$ & \\
\hline Pregnant controls & 15 & 258.4 & & \\
\hline Non-pregnant controls & 10 & 138.7 & & \\
\hline Patients with MM & 78 & 121.7 & & \\
\hline Tumor thickness (mm) & & & $>0.05^{\mathrm{b}}$ & -0.188 \\
\hline T0 (no evidence of primary tumor) & 11 & 129.3 & & \\
\hline $\mathrm{T} 1(\leq 1)$ & 12 & 148.1 & & \\
\hline $\mathrm{T} 2(1.1-2.0)$ & 21 & 102.0 & & \\
\hline T3 $(2.1-4.0)$ & 22 & 136.2 & & \\
\hline $\mathrm{T} 4(>4)$ & 11 & 93.3 & & \\
\hline $\mathrm{S} 100$ calcium-binding protein $\mathrm{B}, \mu \mathrm{g} / \mathrm{l}$ & & & $>0.05^{\mathrm{c}}$ & -0.049 \\
\hline$<0.105$ & 57 & 124.4 & & \\
\hline$\geq 0.105$ & 13 & 111.9 & & \\
\hline Lactate dehydrogenase, U/l & & & $>0.05^{\mathrm{c}}$ & -0.048 \\
\hline$<248$ & 46 & 128.1 & & \\
\hline$\geq 248$ & 24 & 110.5 & & \\
\hline Clinical stage & & & $>0.05^{\mathrm{b}}$ & -0.042 \\
\hline I & 7 & 113.6 & & \\
\hline II & 12 & 122.2 & & \\
\hline III & 25 & 132.2 & & \\
\hline IV & 34 & 116.8 & & \\
\hline
\end{tabular}

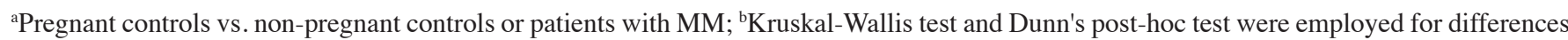
between multiple groups; ' Wilcoxon Mann-Whitney U test was used for differences between two groups. 2-ME, 2-methoxyestradiol.

Although a relatively large number of 78 patients with malignant melanoma in different tumour stages were examined in this study, the tumour subgroups were in part relatively small, so that a further subdivision was not possible. Since no trend could be derived from the analyses so far, it is likely, that further subgroup analyses would not have yielded any additional findings. A further limitation is that the serum levels were determined only at one time point of the disease, regardless of the state of treatment. Future studies should address several time points of the disease and additionally evaluate treatments and tumour burden in order to rule out possible confounders.

In conclusion, these results are the first to indicate that endogenous 2-ME serum levels are not reduced in patients with MM. Therefore, in contrast to the potential treatment effects of exogenously administered 2-ME, endogenous 2-ME serum levels cannot be used as diagnostic or prognostic marker in MM. In our opinion, further investigations should focus on the therapeutic applications of systemically administered 2-ME in patients with $\mathrm{MM}$, rather than the prognostic potential of 2-ME serum levels.

\section{Acknowledgements}

Not applicable.

\section{Funding}

No funding was received.

\section{Availability of data and materials}

The datasets generated and/or analyzed during the current study are available from the corresponding author on reasonable request.

\section{Authors' contributions}

IH, RK, SK, CL, FL and MM conceived and designed the experiments. IH, JK, NZ and MD performed the experiments. IH, JK and FW analysed the data. MD, IH, JK, FW, SK, CL, NZ and FL contributed to data/materials/analysis tools. IH, JK and MM assessed the authenticity of the raw data and wrote the paper. All authors have read and approved the final version of the manuscript.

\section{Ethics approval and consent to participate}

All patients and healthy controls in the present study provided written informed consent prior to enrolment and publication of any data according to the principles outlined in the Declaration of Helsinki. The study protocol was approved by the Institutional Ethics Committee Frankfurt (ethic vote SDO-01-2013) and Mainz [ethic vote 837.270.14 (9509)].

\section{Patient consent for publication}

Not applicable. 


\section{Competing interests}

The authors declare that they have no competing interests.

\section{References}

1. Barnes B, Kraywinkel K, Nowossadeck E, Schönfeld I, Starker A, Wienecke A and Wolf U: Bericht zum Krebsgeschehen in Deutschland 2016. Robert Koch-Institut, Berlin, 2016.

2. Malissen N and Grob JJ: Metastatic melanoma: Recent therapeutic progress and future perspectives. Drugs 78: 1197-1209, 2018.

3. Amin MB, Edge S, Greene F, Byrd DR, Brookland RK, Washington MK, Gershenwald JE, Compton CC, Hess KR, Sullivan DC (eds), et al: AJCC Cancer Staging Manual. Springer International Publishing, New York, NY, 2017.

4. Mocellin S,Zavagno G and Nitti D: The prognostic value of serum S100B in patients with cutaneous melanoma: A meta-analysis. Int J Cancer 123: 2370-2376, 2008.

5. Karagiannis P, Fittall $\mathrm{M}$ and Karagiannis SN: Evaluating biomarkers in melanoma. Front Oncol 4: 383, 2014.

6. Perez-Sepulveda A, Espana-Perrot PP, Norwitz ER and Illanes SE: Metabolic pathways involved in 2-methoxyestradiol synthesis and their role in preeclampsia. Reprod Sci 20 1020-1029, 2013.

7. Shen Z, Wu Y, Chen X, Chang X, Zhou Q, Zhou J, Ying H, Zheng J, Duan T and Wang K: Decreased maternal serum 2-methoxyestradiol levels are associated with the development of preeclampsia. Cell Physiol Biochem 34: 2189-2199, 2014.

8. Parada-Bustamante A, Valencia C, Reuquen P, Diaz P, RincionRodriguez R and Orihuela PA: Role of 2-methoxyestradiol, an endogenous estrogen metabolite, in health and disease. Mini Rev Med Chem 15: 427-438, 2015.

9. Huang XX, Wang RX, Lin Q, Chen W, Pan Y, Wang SQ, Weng ZL and Huang WP: Inhibitory effects of 2-methoxyestradiol on cell growth and invasion in human bladder cancer T-24 cells. Pharmazie 72: 87-90, 2017.
10. Zhe N, Chen S, Zhou Z, Liu P, Lin X, Yu M, Cheng B, Zhang Y and Wang J: HIF-1 $\alpha$ inhibition by 2 -methoxyestradiol induces cell death via activation of the mitochondrial apoptotic pathway in acute myeloid leukemia. Cancer Biol Ther 17: 625-634, 2016.

11. Massaro RR, Faiao-Flores F, Rebecca VW, Sandri S, AlvesFernandes DK, Pennacchi PC, Smalley KS and Maria-Engler SS: Inhibition of proliferation and invasion in 2D and 3D models by 2 -methoxyestradiol in human melanoma cells. Pharmacol Res 119: 242-250, 2017.

12. Kato S, Sadarangani A, Lange S, Delpiano AM, Vargas M, Brañes J, Carvajal J, Lipkowitz S, Owen GI and Cuello MA: 2-methoxyestradiol mediates apoptosis through caspase-dependent and independent mechanisms in ovarian cancer cells but not in normal counterparts. Reprod Sci 15: 878-894, 2008.

13. Ricker JL, Chen Z, Yang XP, Pribluda VS, Swartz GM and Van Waes C: 2-methoxyestradiol inhibits hypoxia-inducible factor 1alpha, tumor growth, and angiogenesis and augments paclitaxel efficacy in head and neck squamous cell carcinoma. Clin Cancer Res 10: 8665-8673, 2004.

14. El Naga RN, El-Demerdash E, Youssef SS, Abdel-Naim AB and El-Merzabani M: Cytotoxic effects of 2-methoxyestradiol in the hepatocellular carcinoma cell line HepG2. Pharmacology 84: 9-16, 2009.

15. Solum EJ,Akselsen OW, Vik A and Hansen TV: Synthesis and pharmacological effects of the anti-cancer agent 2-methoxyestradiol. Curr Pharm Des 21: 5453-5466, 2015.

16. Zhao H, Jiang Y, Liu Y, Yun C and Li L: Endogenous estrogen metabolites as biomarkers for endometrial cancer via a novel method of liquid chromatography-mass spectrometry with hollow fiber liquid-phase microextraction. Horm Metab Res 47: 158-164, 2015.

17. Dobos J, Timar J, Bocsi J, Burián Z, Nagy K, Barna G, Peták I and Ladányi A: In vitro and in vivo antitumoreffect of 2 -methoxyestradiol on human melanoma. Int J Cancer 112: 771-776, 2004.

18. Newman SP, Leese MP, Purohit A, James DR, Rennie CE, Potter BV and Reed MJ: Inhibition of in vitro angiogenesis by 2-methoxy- and 2-ethyl-estrogen sulfamates. Int J Cancer 109: $533-540,2004$ 